ARTICLE

\title{
Local-electrostatics-induced oxygen octahedral distortion in perovskite oxides and insight into the structure of Ruddlesden-Popper phases
}

\author{
Youngjae Hong ${ }^{1}$, Pilgyu Byeon (1) 1, Jumi Bak1, Yoon Heo (1) 1, Hye-Sung Kim¹, Hyung Bin Bae ${ }^{2}$ \& \\ Sung-Yoon Chung ${ }^{1 凶}$
}

As the physical properties of $\mathrm{ABX}_{3}$ perovskite-based oxides strongly depend on the geometry of oxygen octahedra containing transition-metal cations, precise identification of the distortion, tilt, and rotation of the octahedra is an essential step toward understanding the structure-property correlation. Here we discover an important electrostatic origin responsible for remarkable Jahn-Teller-type tetragonal distortion of oxygen octahedra during atomiclevel direct observation of two-dimensional $[A X]$ interleaved shear faults in five different perovskite-type materials, $\mathrm{SrTiO}_{3}, \mathrm{BaCeO}_{3}, \mathrm{LaCoO}_{3}, \mathrm{LaNiO}_{3}$, and $\mathrm{CsPbBr}_{3}$. When the $[A X]$ sublayer has a net charge, for example $[\mathrm{LaO}]^{+}$in $\mathrm{LaCoO}_{3}$ and $\mathrm{LaNiO}_{3}$, substantial tetragonal elongation of oxygen octahedra at the fault plane is observed and this screens the strong repulsion between the consecutive $[\mathrm{LaO}]^{+}$layers. Moreover, our findings on the distortion induced by local charge are identified to be a general structural feature in lanthanide-based $\mathrm{A}_{n+1} \mathrm{~B}_{n} \mathrm{X}_{3 n+1}$-type Ruddlesden-Popper (RP) oxides with charged [LnO $]^{+}$( $\mathrm{Ln}=\mathrm{La}, \mathrm{Pr}, \mathrm{Nd}, \mathrm{Eu}$, and $\mathrm{Gd}$ ) sublayers, among more than $80 \mathrm{RP}$ oxides and halides with high symmetry. The present study thus demonstrates that the local uneven electrostatics is a crucial factor significantly affecting the crystal structure of complex oxides.

\footnotetext{
${ }^{1}$ Department of Materials Science and Engineering and KAIST Institute for the Nanocentury, Korea Advanced Institute of Science and Technology, Yuseonggu, Daejeon 34141, Korea. ${ }^{2}$ KAIST Analysis Center, Korea Advanced Institute of Science and Technology, Yuseong-gu, Daejeon 34141 , Korea.

凶email: sychung@kaist.ac.kr
} 
T he crystal structure of $\mathrm{ABO}_{3}$-type perovskites is based on the geometric stability of packing for ions of different sizes and valence states. While the relatively large cation $\mathrm{A}$ is coordinated with 12 oxygen anions at the dodecahedral sites, the smaller cation B is located in the oxygen octahedra connected to each other by corner-sharing, agreeing well with Pauling's first rule for ionic compounds ${ }^{1}$. In particular, the distortion, including tilt and rotation, of oxygen octahedra is known to be a crucial factor governing the dielectric, magnetic, optical, and catalytic properties of perovskites ${ }^{2,3}$. As a consequence, the direct identification and description of the geometry of oxygen octahedra in perovskites and derivative oxides have been key issues over the last decade ${ }^{4-10}$.

In addition to the relative ionic size and the cation-anion bond strength, a significant electronic contribution to the structural distortion in oxides has been noted as the Jahn-Teller effect ${ }^{11,12}$. The central argument of this effect is that the energy of $d$-orbital electrons of transition-metal ions can be significantly lowered by spontaneous tetragonal elongation in such a way as to remove the degeneracy of the $d$-orbital levels. As is already known very well, high-spin $\mathrm{Mn}^{3+}\left(3 d^{4}\right)$ and low-spin $\mathrm{Cu}^{2+}\left(3 d^{9}\right)$ are typical Jahn-Teller active cations, demonstrating that the splitting of the degenerate $d_{x 2-y 2}$ and $d_{z 2}$ levels of the $e_{g}$ subshells and subsequent energy stabilization of electrons is achieved via substantial tetragonal distortion of oxygen octahedra. A key message of the Jahn-Teller effect is that the electronic structure of transitionmetal ions should be taken into account to fully understand the crystal (or molecular) structure.

Recently, intriguing local distortion of oxygen octahedra was reported in heteroepitaxial $\mathrm{LaNiO}_{3}$ thin films ${ }^{13}$. Even though this nickelate has a pseudocubic structure, tetragonal elongation of $\left\{\mathrm{NiO}_{6}\right\}$ octahedra was identified to take place exclusively at the planes of Ruddlesden-Popper (RP) two-dimensional shear faults. Taking into account that the shear faults, consisting of consecutive $[\mathrm{LaO}]^{+}$layers, have electrostatically repulsive instability, the protrusion of negatively charged oxygen anions toward the shear plane was reasonably claimed to be energetically favorable for alleviating the strong repulsion between the $[\mathrm{LaO}]^{+}$layers. Although this previous study was confined to a single material, $\mathrm{LaNiO}_{3}$, provided a physically sound explanation, further extensive work remains necessary in order to clarify and generalize the local-charge hypothesis as another important electrostatic origin responsible for the remarkable tetragonal distortion of oxygen octahedra in perovskite-derivative oxides.

To this end, in this work, we extended our atomic-scale direct observations of two-dimensional shear faults ${ }^{14-18}$ into five different perovskite-type materials, $\mathrm{SrTiO}_{3}, \mathrm{BaCeO}_{3}, \mathrm{LaCoO}_{3}$, $\mathrm{LaNiO}_{3}$, and $\mathrm{CsPbBr}_{3}$. First, we observed substantial tetragonal distortion of $\left\{\mathrm{BO}_{6}\right\}$ octahedra at the fault plane, when an interleaved $[\mathrm{AO}]-[\mathrm{AO}]$ shear fault has a local charge (for example, $[\mathrm{LaO}]^{+}$in $\mathrm{LaCoO}_{3}$ and $\mathrm{LaNiO}_{3}$ ). In stark contrast, no significant structural distortion was probed in the $\left\{\mathrm{BO}_{6}\right\}$ octahedra when the $[\mathrm{AO}]$ sublayer has no effective charge $\left([\mathrm{SrO}]\right.$ in $\mathrm{SrTiO}_{3},[\mathrm{BaO}]$ in $\mathrm{BaCeO}_{3}$, and $[\mathrm{CsBr}]$ in $\mathrm{CsPbBr}$ ). Furthermore, by examining a series of $87 \mathrm{~A}_{n+1} \mathrm{~B}_{n} \mathrm{X}_{3 n+1}$-type Ruddlesden-Popper (RP) oxides and halides with high symmetry, we found that this local-chargeinduced tetragonal elongation of oxygen octahedra is a striking common feature observed in most of the RP phases having the charged [AO] layer. Consequently, our findings on the electrostatic origin of the distortion appear to be a general phenomenon in oxides containing multiple cations.

\section{Results and discussion}

Observation of shear faults. For fault observations, we prepared polycrystalline dense $\mathrm{SrTiO}_{3}, \mathrm{BaCeO}_{3}$, and $\mathrm{LaCoO}_{3}$ sintered pellets, epitaxial $\mathrm{LaNiO}_{3}$ thin films, and $\mathrm{CsPbBr}$ nanocrystals (see the "Methods" section), all of which contain homologous stacking faults represented by two consecutive rocksalt [AX] layers $(\mathrm{A}=\mathrm{Sr}, \mathrm{Ba}, \mathrm{La}, \mathrm{Cs} ; \mathrm{X}=\mathrm{O}, \mathrm{Br})$. Figure 1 shows a series of high-angle annular dark-field (HAADF) scanning transmission electron microscopy (STEM) ${ }^{19-21}$ images at atomic resolution, verifying the presence of rocksalt $[\mathrm{AX}]$ faults in all five perovskite crystals (see Supplementary Fig. 1 for lower magnification images). As the atomic number, $Z$, of $\mathrm{Ba}\left(Z_{\mathrm{Ba}}=56\right)$ and $\mathrm{Ce}\left(Z_{\mathrm{Ce}}\right.$ $=58)$ is very similar, individual columns are not readily discriminated in $\mathrm{BaCeO}_{3}$, although the image contrast of the faults is sufficiently distinguishable at a lower magnification (Supplementary Fig. 1b). A chemical map obtained by electron energyloss spectroscopy (EELS) is thus provided in Fig. 1b to confirm the $[\mathrm{BaO}]-[\mathrm{BaO}]$ layers at the fault. In addition, note that the $Z$ value of $\mathrm{Cs}\left(Z_{\mathrm{Cs}}=55\right)$ is much lower than that of $\mathrm{Pb}\left(Z_{\mathrm{Pb}}=82\right)$ in $\mathrm{CsPbBr}_{3}$. (See Supplementary Fig. 2 for additional STEM
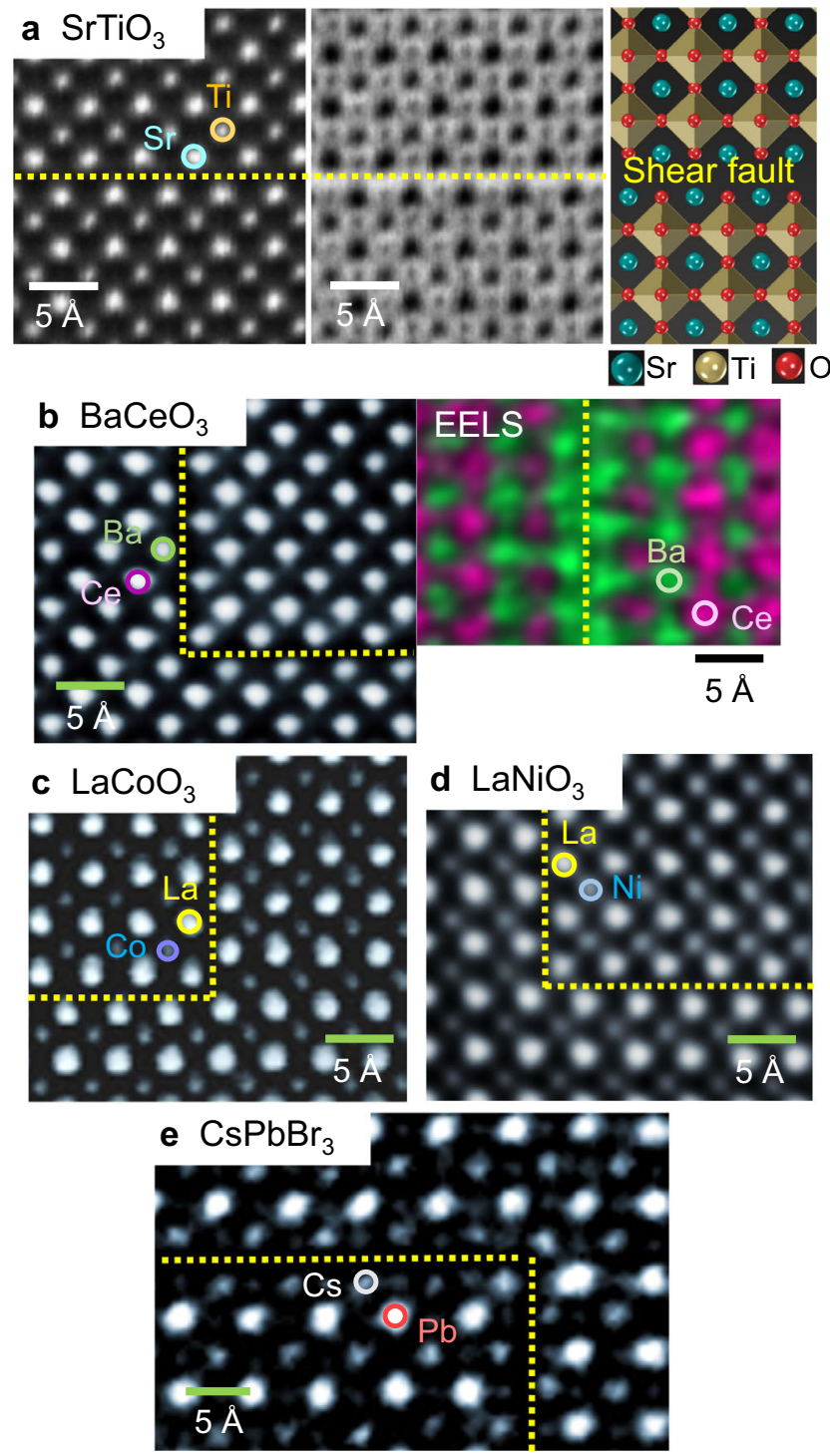

Fig. 1 STEM images for the formation of homologous shear faults. Each image was acquired in a $\mathrm{SrTiO}_{3}$, b $\mathrm{BaCeO}_{3}, \mathbf{c} \mathrm{LaCoO}_{3}$, d $\mathrm{LaNiO}_{3}$, and e $\mathrm{CsPbBr}_{3}$. The yellow broken lines in each image indicate the fault planes consisting of two consecutive $[\mathrm{AX}]$ sublayers, as schematically described in a together with an annular bright-field image. The atomic-scale chemical map shown in $\mathbf{b}$ was obtained by using EELS to verify the consecutive $[\mathrm{BaO}]$ sublayers. 
images of $\mathrm{CsPbBr}_{3}$ nanocrystals). Consequently, the column intensity at the [CsBr] faults in a HAADF image should be distinctively lower, as shown in Fig. 1e, in contrast to the image feature at the faults observed in $\mathrm{SrTiO}_{3}, \mathrm{LaCoO}_{3}$, and $\mathrm{LaNiO}_{3}$.

Oxygen displacement and local electrostatics. To visualize the position of light-element anions, we utilized the annular brightfield $(\mathrm{ABF})$ mode in $\mathrm{STEM}^{22-29}$. All the ABF images acquired from the fault region in the five perovskites are provided in Fig. 2 (see Supplementary Fig. 3 for pairs of ABF and HAADF images of the bulk crystals as references). A readily recognizable structural feature from this set of atomic-scale observations is that oxygen octahedra at the faults are asymmetrically elongated along the $z$ axis in $\mathrm{LaCoO}_{3}$ and $\mathrm{LaNiO}_{3}$ (Fig. 2b), while no distortion of anion octahedra is identified in the other three perovskites, $\mathrm{SrTiO}_{3}$, $\mathrm{BaCeO}_{3}$, and $\mathrm{CsPbBr}_{3}$, as denoted by a white square diamond in each image in Fig. 2a. In particular, more than a $20 \%$ increment in the Co-O length from 1.9 to $2.3 \AA$ in $\mathrm{LaCoO}_{3}$ and a $15 \%$
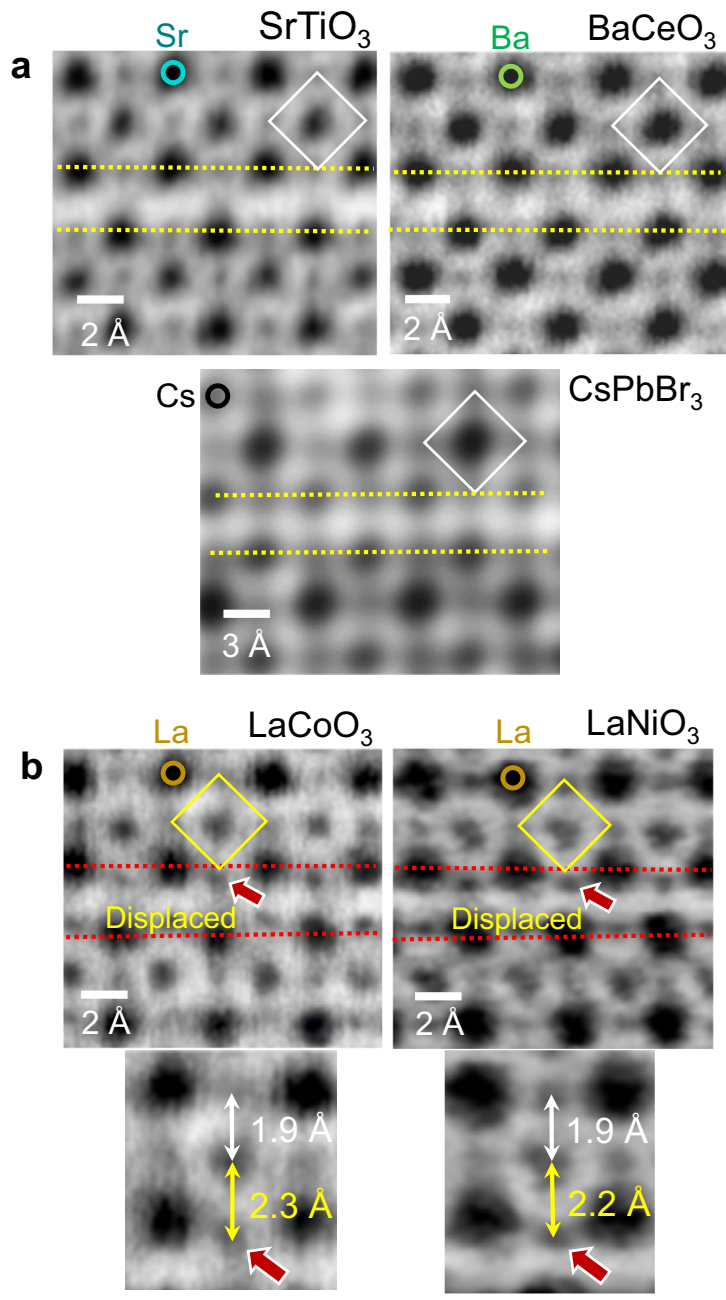

Fig. 2 ABF-STEM images of the shear faults to visualize the position of anions. a As denoted by a white square diamond in each image, no distortion of anion octahedra is observed at the faults (yellow broken lines) in $\mathrm{SrTiO}_{3}, \mathrm{BaCeO}_{3}$, and $\mathrm{CsPbBr}_{3}$. b In contrast, as indicated by a red arrow in each image, substantial displacement of oxygen anions at the fault planes should be noted in $\mathrm{LaCoO}_{3}$ and $\mathrm{LaNiO}_{3}$, showing the position deviation from the yellow square diamonds. Magnified images directly provide quantitative information on the elongation of bond lengths in $\mathrm{Co}-\mathrm{O}(2.3 \AA)$ and $\mathrm{Ni}-\mathrm{O}(2.2 \AA)$. increment in the Ni-O length from 1.9 to $2.2 \AA$ in $\mathrm{LaNiO}_{3}$ are presented in the magnified images in Fig. $2 \mathrm{~b}$.

These two perovskite groups revealing distinct octahedral aspects have their own local electrostatic characteristics from the viewpoint of a layer structure. As schematically depicted in Fig. 3a, no effective local charge is noted in each of the $[\mathrm{AX}]$ and $\left[\mathrm{BX}_{2}\right]$ sublayers in three perovskites, $\mathrm{SrTiO}_{3}, \mathrm{BaCeO}_{3}$, and $\mathrm{CsPbBr}_{3}$. For example, because the valence states of $\mathrm{Sr}, \mathrm{Ti}$, and $\mathrm{O}$ in $\mathrm{SrTiO}_{3}$ are $2+, 4+$, and $2-$, respectively, the sublayers, $[\mathrm{SrO}]^{0}$ and $\left[\mathrm{TiO}_{2}\right]^{0}$, have no net charges ${ }^{30}, 31$. Therefore, no electrostatic perturbation is induced at the homologous [SrO]-[SrO] fault plane, indicating that the (pseudo)cubic bulk structure can be preserved at the fault region without showing elongation of oxygen octahedra. In the same manner, there is no local charge at the faults in $\mathrm{BaCeO}_{3}\left([\mathrm{BaO}]^{0}\right)$ and $\mathrm{CsPbBr}_{3}$ $\left([\mathrm{CsBr}]^{0}\right)$.

In contrast, the La-based perovskite oxides, $\mathrm{LaCoO}_{3}$ and $\mathrm{LaNiO}_{3}$, contain local net charge at each of the sublayers, as described in Fig. 3b. As the valence states of $\mathrm{La}, \mathrm{Co}$ (and Ni), and $\mathrm{O}$ are $3+, 3+$, and $2-$, respectively, the bulk structure of $\mathrm{LaCoO}_{3}$ (and $\mathrm{LaNiO}_{3}$ ) consists of positively charged $[\mathrm{LaO}]^{+}$layers and negatively charged $\left[\mathrm{CoO}_{2}\right]^{-}$(and $\left[\mathrm{NiO}_{2}\right]^{-}$) layers in an alternating manner along the $z$ direction. Consequently, if two $[\mathrm{LaO}]^{+}$layers are consecutively placed at the faults, electrostatically strong repulsion is inevitably induced, resulting in serious local instability (Fig. 3b). One of the easiest way to reduce the positive-charge repulsion between two $[\mathrm{LaO}]^{+}$layers is that the negatively charged $\mathrm{O}^{2-}$ anions adjacent to the fault displace toward the fault plane and thereby screen the effective positive charge. Even if the displacement of oxygen anions is at an angstrom level, it appears to be fairly efficient to significantly reduce the repulsive instability at the fault plane.

Theoretical calculations. The displacement of oxygen anions was also verified by ab initio density functional theory (DFT) calculation. Figure $3 \mathrm{c}$ shows the atom position variation in a $\mathrm{LaNiO}_{3}$ supercell containing a shear fault during the DFT calculation for geometry optimization. As shown in the supercell illustration of the first self-consistent field (SCF) iteration step, the initial configuration of oxygen atoms (light red) at the fault plane was set to be identical to the position of oxygen in the bulk. It is noted that the $\mathrm{Ni}-\mathrm{O}$ bonds adjacent to the fault substantially elongate even after one SCF step, as can be seen in the second illustration, together with significant stabilization of the lattice energy (see Supplementary Fig. 4 for the plot of the lattice energy variation with SCF steps). The third illustration shows the finally optimized configuration of the supercell cell, revealing that the $\mathrm{Ni}-\mathrm{O}$ bond length adjacent to the fault plane is $2.1 \AA$ in the magnified illustration. This value after geometry optimization is in fairly good agreement with the experimentally observed $\mathrm{Ni}-\mathrm{O}$ length, $2.2 \AA$, as directly compared in the ABF-STEM image. The DFT calculation thus confirms that elongation of the $\mathrm{Ni}-\mathrm{O}$ bond length at the fault is an energetically stable configuration. At the same time, $\mathrm{La}^{3+}$ cations are also observed to displace along the $z$ axis out of the fault plane in the series of supercell illustrations in order to facilitate the reduction of the repulsion at the fault.

We also carried out another set of DFT calculations to compare the density of states (DOS) of the Ni $3 d$ and O $2 p$ orbitals in $\mathrm{LaNiO}_{3}$ with and without a fault. As demonstrated in Supplementary Fig. 5, the overall substantial degree of overlap between the $\mathrm{Ni} 3 d$ and $\mathrm{O} 2 p$ states in addition to the metallic behavior with no bandgap does not significantly vary between the two DOS plots. However, as indicated by arrows, the noticeable increases of $\mathrm{O} 2 p$ and $\mathrm{Ni} 3 d$ states at the bottom, in the middle, and at the top of the band in the DOS plot are key changes of the 

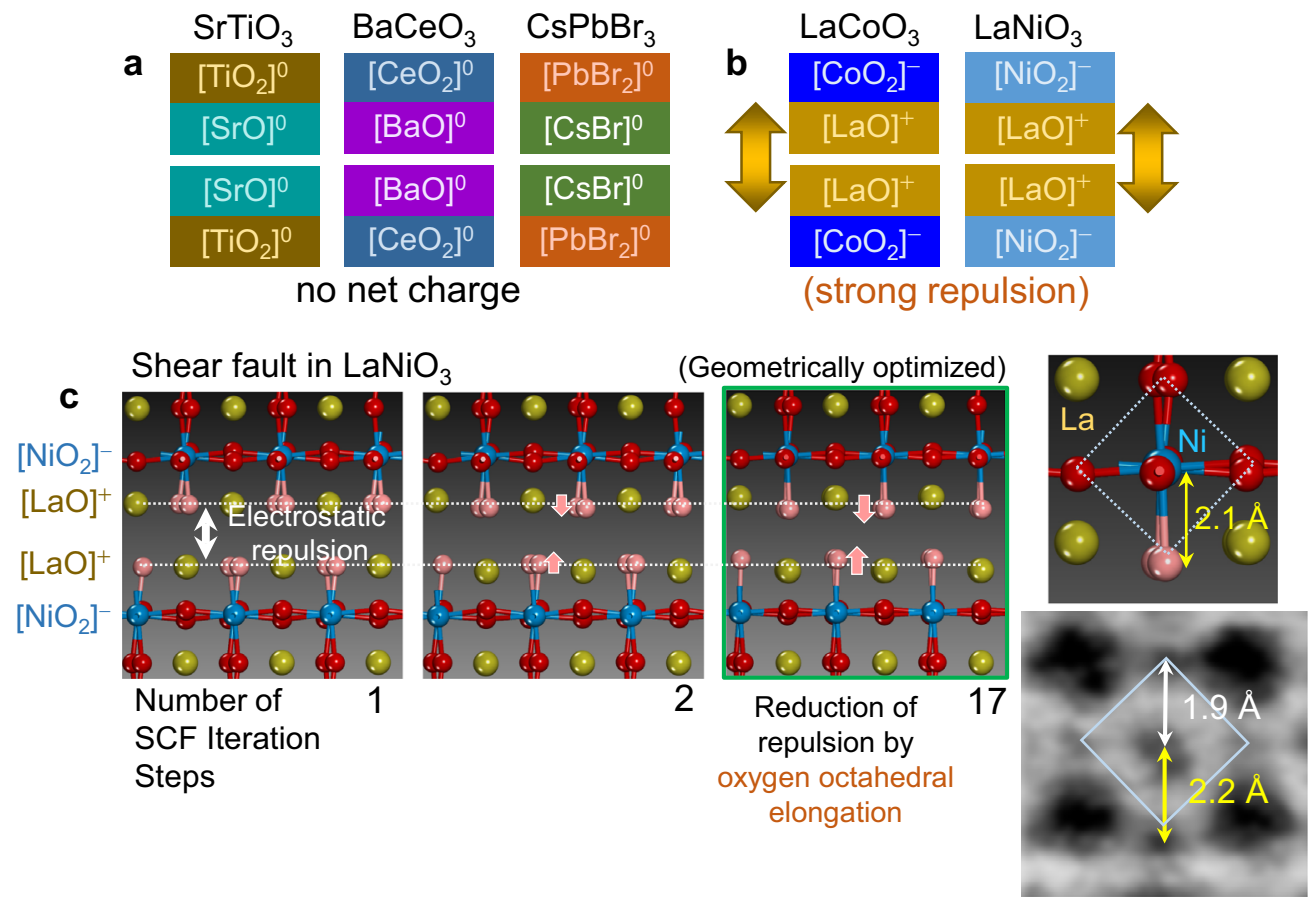

Fig. 3 Description of sublayer polarity in perovskites and geometry optimization of atom configuration during DFT calculation. The effective charges of $[\mathrm{AX}]$ and $\left[\mathrm{BX}_{2}\right]$ sublayers in the five perovskites are indicated. a No polarity of sublayers is present in $\mathrm{SrTiO}_{3}, \mathrm{BaCeO}_{3}$, and $\mathrm{CsPbBr}_{3}$. $\mathbf{b}$ In contrast, $[\mathrm{LaO}]+$ in $\mathrm{LaCoO}_{3}$ and $\mathrm{LaNiO}_{3}$ is noted to contain strong electrostatic repulsion at the fault. $\mathbf{c}$ Geometry optimization with $\mathrm{SCF}$ steps at the fault in $\mathrm{LaNiO}$ is achieved by the oxygen displacements toward the fault plane, as denoted by small arrows in the illustrations. Good agreement of the $\mathrm{Ni}-\mathrm{O}$ elongation obtained by the DFT calculation and the direct ABF-STEM observation is verified.

electronic structure that result from the $z$ axis elongation of $\left[\mathrm{NiO}_{6}\right]$ octahedra at the fault plane. When six oxygen ligands around a metal cation are located in an octahedral symmetry, the metal $3 d$ states are discreetly split as two degenerate $t_{2 \mathrm{~g}}$ and $e_{\mathrm{g}}$ levels. In contrast, if this symmetry is broken either by oxygen octahedral elongation or via other perturbation $27,28,32,33$, the degeneracy of the two levels is removed, resulting in a wide range of energy levels of the metal $3 d$ states in addition to the $\mathrm{O} 2 p$ states for hybridization.

EELS analyses. Atomic-column resolved EELS analyses on the RP shear faults were performed to examine the variation of energyloss spectra in addition to the oxidation state of transition metals. As the $\mathrm{Ni}-\mathrm{L}_{3}$ edge seriously overlaps with the $\mathrm{La}-\mathrm{M}_{4}$ edge, we selected $\mathrm{LaCoO}_{3}$ and $\mathrm{SrTiO}_{3}$ samples containing a shear fault instead of $\mathrm{LaNiO}_{3}$ for the EELS analyses. Figure 4 shows a comparison of Co-L and $\mathrm{O}-\mathrm{K}$ edges acquired from the bulk and the fault in $\mathrm{LaCoO}_{3}$. First, no noticeable changes of the peak intensity and position in the Co- $\mathrm{L}_{2,3}$ edges from the bulk and the fault were identified, directly indicating the oxidation state of $\mathrm{Co}^{3+}$ is invariant at the fault. Second, the three major peaks in the $\mathrm{O}-\mathrm{K}$ edge are represented for the hybridizations of $\mathrm{O} 2 p$ with $\mathrm{Co}$ $3 d$ (peak A), La $5 d$ (peak B) and Co $4 s / 4 p$ (peak C), respectively ${ }^{34-37}$. The intensity of peak A obtained from the fault substantially diminishes, as denoted by a red arrow in the plot, while the intensity of the other peaks does not significantly vary between the bulk and the fault. This reduction of the pre-peak in the $\mathrm{O}-\mathrm{K}$ edge appears to consistently correlate with the elongation of the $\mathrm{Co}-\mathrm{O}$ bond length at the faults. Because the bond distance between $\mathrm{Co}$ and $\mathrm{O}$ becomes longer, the effect of oxygen ligands on Co should be reduced, and thereby the degree of hybridization of $\mathrm{O} 2 p$ with Co $3 d$ orbitals decreases. Consequently, as demonstrated in a perovskite cobaltate ${ }^{35}$ and layered lithium transition-metal oxides ${ }^{38,39}$, which contain oxygen vacancies, the
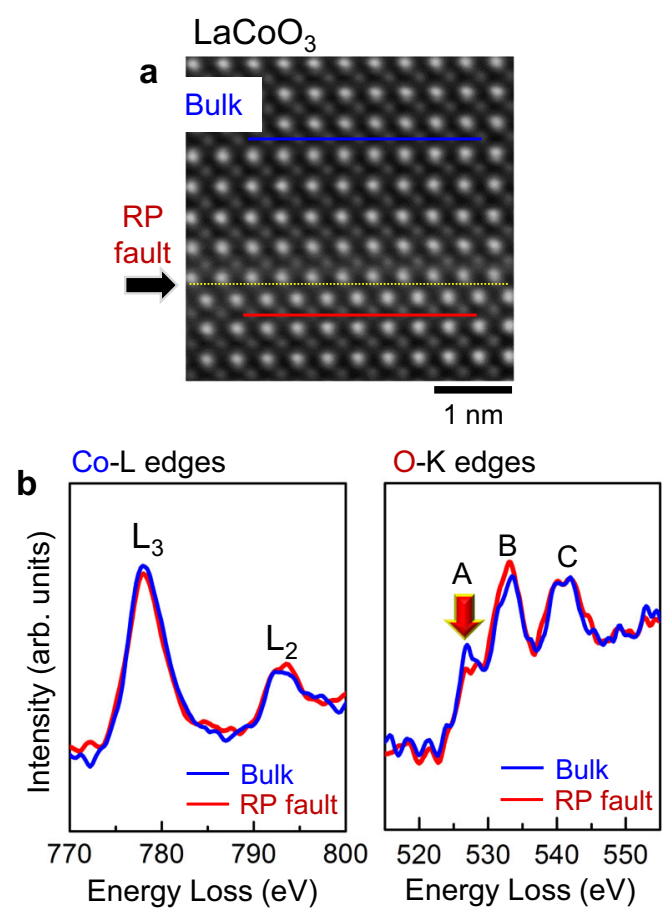

Fig. 4 EELS analysis at the RP fault in $\mathrm{LaCoO}_{3}$. a As indicated in the image, Co- $L$ and $O-K$ edges were acquired from the bulk (blue line) and the RP fault (red line) in $\mathrm{LaCoO}_{3}$. b Three major peaks in the $\mathrm{O} K$-edges represent the hybridizations of $\mathrm{O} 2 p$ with Co $3 d(A)$, La $5 d(B)$, and Co $4 s / 4 p(C)$ orbitals, respectively. No noticeable peak shift or intensity variation is observed in the Co- $L$ edges, showing the invariant oxidation states of $\mathrm{Co}^{3+}$ at the fault plane. $A$ red arrow in the plot of $\mathrm{O}-\mathrm{K}$ edges indicates the intensity reduction of peak $A$ at the fault (red curves), whereas the intensities of peaks $B$ and $C$ hardly vary between the bulk and the fault. 
lower intensity of peak A likely stems from the comparatively weak orbital interaction. Indeed, such a peak reduction was not observable in the O-K edge obtained from the fault in $\mathrm{SrTiO}_{3}$, where no elongation of $\mathrm{Ti}-\mathrm{O}$ bond length is identified at the faults (see Supplementary Fig. 6 for details on the EELS analysis of the fault in $\mathrm{SrTiO}_{3}$ ).

Local structures of RP oxides. In addition to directly demonstrating the distortion of oxygen octahedra at the faults in perovskite oxides, the findings in this work have further notable implications for the in-depth understanding of the structures of a variety of RP oxides and halides, which exhibit a very wide range of physical properties encompassing dielectric and unusual ferroic, multiferroic, electrocatalytic, and optical behavior ${ }^{7-10,40-45}$. As can be easily recognized from their general formula, $\mathrm{A}_{n+1}$ $\mathrm{B}_{n} \mathrm{X}_{3 n+1}$ or equivalently $\mathrm{AX}\left[\mathrm{ABX}_{3}\right]_{n}$, the presence of consecutive [AX] sublayers is a common structural feature irrespective of the value of $n$. In particular, it is noted that every anion octahedron in layer-structured $\mathrm{A}_{2} \mathrm{BX}_{4}(n=1)$ (or $\mathrm{AX}\left[\mathrm{ABX}_{3}\right]$ ) is in contact with two shear planes, as denoted by yellow broken lines in the atomic illustration of $\mathrm{Sr}_{2} \mathrm{TiO}_{4}$ in Fig. 5a. If the [AX] layer has an effective charge, as aforementioned in $[\mathrm{LaO}]^{+}$, charge-induced electrostatic repulsion would considerably accumulate over the entire crystal lattice of $\mathrm{A}_{2} \mathrm{BX}_{4}$. Consequently, substantial elongation of $\left\{\mathrm{BX}_{6}\right\}$ octahedra should take place to significantly reduce the repulsive instability in the consecutive [AX] layers.

We examined more than 80 RP-type oxides and halides with high symmetry from the open database of inorganic materials (the Materials Project) and the Inorganic Crystal Structure Database (ICSD) to scrutinize whether the local net charge of [AX] sublayers exerts a general influence on the tetragonal distortion of anion octahedra in RP phases. Note that most of the $\mathrm{RP}$ oxides and halides under our examination in this work were confined to centrosymmetric phases with a single composition at each of the A and B sites in order to exclude any other complexity originating from the polarity induced by either cation offcentering from the second-order Jahn-Teller effect ${ }^{46,47}$, octahedral rotations $s^{5-7}, 10$, or cation ordering 48,49 . Taking this into consideration, structure comparisons were made largely between RP phases with a space group, $I 4 / \mathrm{mmm}^{9}, 49$.

Supplementary Tables 1-3 list all the structural information regarding the ratios of $x$ axis and $z$ axis $\mathrm{B}-\mathrm{X}$ bond lengths, $L_{z} / L_{x}$, in $87 \mathrm{RP}$ oxides and halides (see the detailed illustration in Supplementary Table 1 for the definition of $L_{z} / L_{x}$ ); Ca-, Sr-, and Ba-based RP oxides with no local charge in Supplementary Table 1, K- and Cs-based RP halides in Supplementary Table S2, and lanthanide-based RP oxides in Supplementary Table 3. "2.1.4.", “3.2.7.", and "4.3.10." in each table represent $\mathrm{A}_{2} \mathrm{BX}_{4}$ $(n=1), \quad \mathrm{A}_{3} \mathrm{~B}_{2} \mathrm{X}_{7} \quad(n=2)$, and $\mathrm{A}_{4} \mathrm{~B}_{3} \mathrm{X}_{10} \quad(n=3) \quad \mathrm{RP}$ phases, respectively. All of the 87 crystal structures are also illustrated in Supplementary Figs. 7-60. This series of tables and supplementary figures demonstrates the notable and exclusive effect of the [AX] local charge on the $z$ axis elongation of octahedra $\left(L_{z} / L_{x}>1.04\right)$ in the cases of lanthanide-based $(\mathrm{A}=\mathrm{La}$, $\mathrm{Pr}, \mathrm{Nd}, \mathrm{Eu}$, and Gd) RP oxides. We set the criterion of $4 \%$ elongation by rounding down the minimum value, 1.048 (which corresponds to $4.8 \%$ elongation in the case of $\mathrm{EuCoO}_{3}$ in

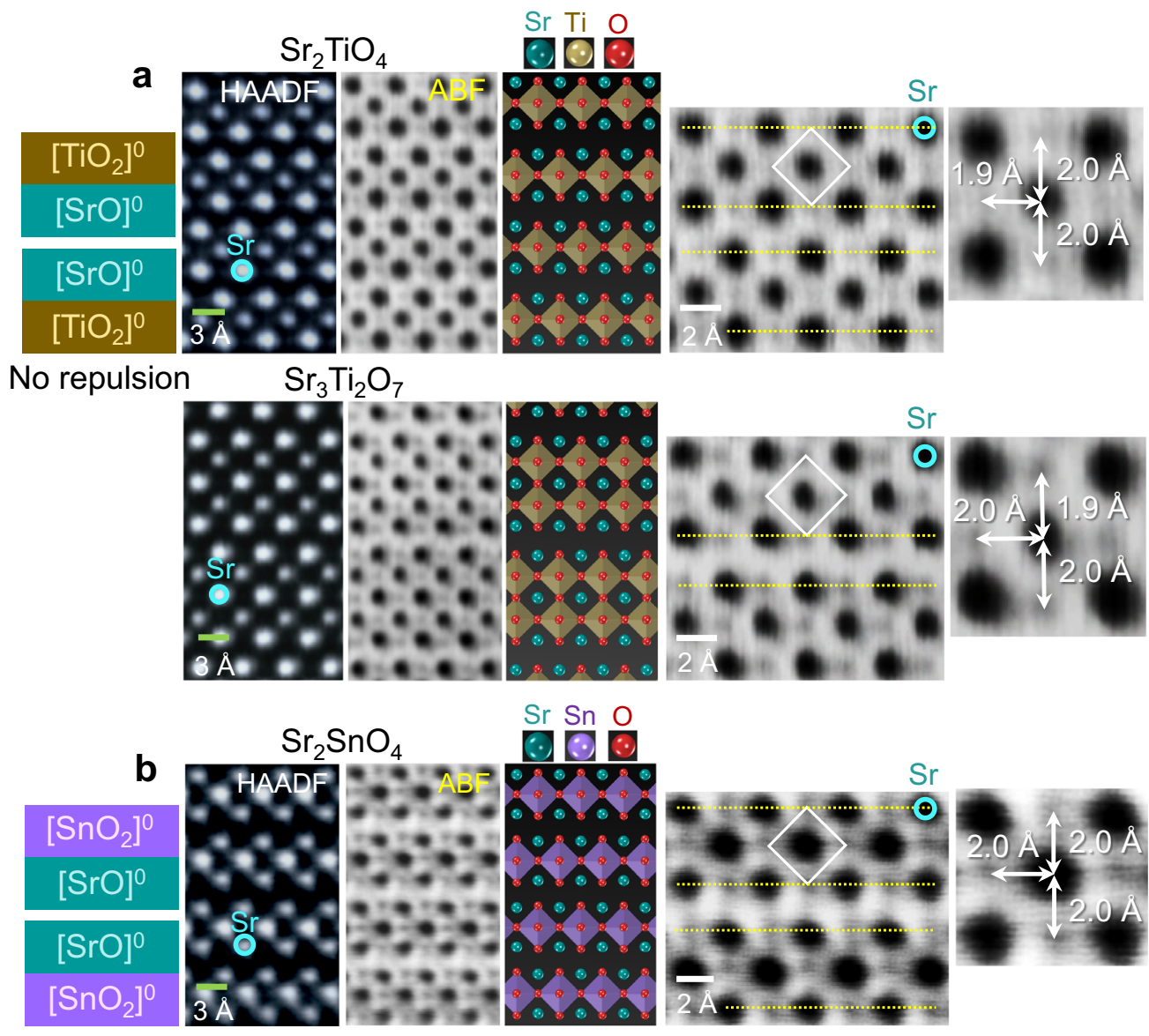

Fig. 5 STEM images showing the structure of Sr-based RP oxides. Atomic-column-resolved images were acquired in a $\mathrm{Sr}_{2} \mathrm{TiO}_{4}$ and $\mathrm{Sr}_{3} \mathrm{Ti}_{2} \mathrm{O}_{7}$ and $\mathbf{b}$ $\mathrm{Sr}_{2} \mathrm{SnO}_{4}$, as listed in Supplementary Table 1. Because these RP oxides have no effective charge in [SrO] sublayers, no distortion of oxygen octahedra is identified in each structure. Magnified ABF images directly provide quantitative information on the Ti-O and $\mathrm{Sn}-\mathrm{O}$ bond lengths. 
Supplementary Table 3), to three decimal places. As denoted by the red-brown background in Supplementary Table 3, all the Lnbased RP oxides exhibit more than $4 \%$ substantial elongation of $z$ axis $\mathrm{B}-\mathrm{O}$ bond lengths, showing strong tetragonal distortion of octahedra (see Supplementary Figs. 46-60 for their crystal structures).

Direct observations in RP oxides. To experimentally visualize the local-charge-induced elongation of oxygen octahedra in addition to referencing the structural database, we carried out direct atomic-column-resolved ABF-STEM observations in a variety of RP oxides selected from Supplementary Tables 1 and 3. Figure 5 exemplifies $\mathrm{ABF}$ images and their enlargements of three Sr-based RP oxides, $\mathrm{Sr}_{2} \mathrm{TiO}_{4}, \mathrm{Sr}_{3} \mathrm{Ti}_{2} \mathrm{O}_{7}$, and $\mathrm{Sr}_{2} \mathrm{SnO}_{4}$ (see Supplementary Figs. 61 for low-magnification STEM images), with no local charge at the fault plane of $[\mathrm{SrO}]^{0}-[\mathrm{SrO}]^{0}$ consecutive layers, as listed in Supplementary Table 1 . The nearly identical $x$ axis and $z$ axis Ti-O (and Sn-O) bond lengths $\left(L_{z}\right.$ and $\left.L_{x}\right)$ in each oxide agree well with the information provided in the table, verifying the ratio $L_{z} / L_{x} \approx 1$.

Figure 6 shows the actual structures and their atomic-scale $\mathrm{ABF}$ images of five lanthanide-based $\mathrm{RP}$ nickelates, $\mathrm{La}_{2} \mathrm{NiO}_{4}$, $\mathrm{La}_{3} \mathrm{Ni}_{2} \mathrm{O}_{7}, \mathrm{La}_{4} \mathrm{Ni}_{3} \mathrm{O}_{10}, \mathrm{Nd}_{2} \mathrm{NiO}_{4}$, and $\mathrm{Pr}_{2} \mathrm{NiO}_{4}$, selected from Supplementary Table 3, with an effective local charge of the sublayers, $[\mathrm{LaO}]^{+},[\mathrm{NdO}]^{+}$, and $[\mathrm{PrO}]^{+}$, as schematically illustrated in the left-hand column (see Supplementary Figs. 62 for low-magnification STEM images). In particular, Fig. 6b, c specifically exemplify the tetragonal elongation of every oxygen octahedra in $\mathrm{Nd}_{2} \mathrm{NiO}_{4}$, and $\mathrm{Pr}_{2} \mathrm{NiO}_{4}$, in addition to $\mathrm{La}_{2} \mathrm{NiO}_{4}$ in Fig. 6a. The Ni-O bond lengths in each RP nickelate can be directly acquired from the ABF images in the right-hand column. As denoted by yellow arrows in the enlargements, the substantial elongation (more than 15\%) of $z$ axis $\mathrm{Ni}-\mathrm{O}$ bonds toward the fault planes is readily recognized. This structural feature is consistently confirmed in La-based RP cobaltates and cuprates, as demonstrated in Fig. 7. Moreover, $\mathrm{Cu}^{2+}$ is a well-known strong Jahn-Teller cation. As a consequence, more prominent $z$ axis elongation $(>27 \%)$ of $\left\{\mathrm{CuO}_{6}\right\}$ octahedra in $\mathrm{La}_{2} \mathrm{CuO}_{4}$ (Fig. 7b) than that of $\left\{\mathrm{CoO}_{6}\right\}$ in $\mathrm{La}_{2} \mathrm{CoO}_{4}$ and $\left\{\mathrm{NiO}_{6}\right\}$ in $\mathrm{La}_{2} \mathrm{NiO}_{4}$ is identified by a combination of the $[\mathrm{LaO}]^{+}$local charge and the Jahn-Teller effect. To clarify that this $>27 \%$ strong elongation of $\left\{\mathrm{CuO}_{6}\right\}$ octahedra is a common aspect in $\mathrm{Pr}_{2} \mathrm{CuO}_{4}$ and $\mathrm{Nd}_{2} \mathrm{CuO}_{4}$ as well (Supplementary Table 3), an additional structure comparison is provided in Supplementary Fig. 63.

We summarize several representative $\mathrm{A}_{2} \mathrm{BX}_{4}(n=1)$ oxides and fluorides among the RP phases with the space group $I 4 / \mathrm{mmm}$ in Supplementary Fig. 64 to highlight the impact of the net charge of the sublayers on the significant elongation of oxygen octahedra. As schematically described in Supplementary Fig. 64a, [SrO] in $\mathrm{Sr}_{2} \mathrm{TiO}_{4},[\mathrm{KF}]$ in $\mathrm{K}_{2} \mathrm{NiF}_{4}$, and [CsF] in $\mathrm{Cs}_{2} \mathrm{CuF}_{4}$ have no effective charge. The six $\mathrm{B}-\mathrm{X}$ bond lengths in each of $\left\{\mathrm{TiO}_{6}\right\},\left\{\mathrm{NiF}_{6}\right\}$, and $\left\{\mathrm{CuF}_{6}\right\}$ octahedra are thus nearly identical $(<4 \%$ variation), showing no substantial geometric distortion. In contrast, as the $[\mathrm{LaO}]^{+}$sublayers in all the La-based RP oxides are positively charged, the protrusion of oxygen anions toward the shear planes is identified and this reduces the repulsive electrostatic instability. Even though $\mathrm{B}$-site cations are the same $\left(\mathrm{Ni}^{2+}\right.$ in $\mathrm{K}_{2} \mathrm{NiF}_{4}$ and $\mathrm{La}_{2} \mathrm{NiO}_{4}$ and $\mathrm{Cu}^{2+}$ in $\mathrm{Cs}_{2} \mathrm{CuF}_{4}$ and $\mathrm{La}_{2} \mathrm{CuO}_{4}$, respectively), the distinct elongation of $\left\{\mathrm{NiO}_{6}\right\}$ in $\mathrm{La}_{2} \mathrm{NiO}_{4}$ and $\left\{\mathrm{CuO}_{6}\right\}$ in $\mathrm{La}_{2} \mathrm{CuO}_{4}$ in contrast to $\left\{\mathrm{NiF}_{6}\right\}$ in $\mathrm{K}_{2} \mathrm{NiF}_{4}$ and $\left\{\mathrm{CuF}_{6}\right\}$ in $\mathrm{Cs}_{2} \mathrm{CuF}_{4}$ clarifies the crucial influence of the electrostatic charge on the tetragonal distortion of oxygen octahedra in RP oxides. Similarly, because the other lanthanide cations, $\mathrm{Ln}=\mathrm{Pr}, \mathrm{Nd}, \mathrm{Gd}$, at the A sites in $\mathrm{RP}$ oxides are trivalent, the $[\mathrm{LnO}]^{+}$sublayers are also positively charged, as in the case of the La-based RP oxides. As specifically exemplified in Supplementary Fig. 64c for $\mathrm{Pr}$ - and $\mathrm{Nd}$-based cobaltates, therefore, substantial $z$ axis octahedral elongation is consistently identified.

When the structure of $\mathrm{A}$-site solid solution $\left(\mathrm{A}^{\prime} \mathrm{A}^{\prime \prime}\right)_{2} \mathrm{BO}_{4}$, (where the valence states of $\mathrm{A}^{\prime}$ and $\mathrm{A}^{\prime \prime}$ are $3+$ and $2+$, respectively) is scrutinized, the effect of electrostatic charge on the elongation of oxygen octahedra is further supported. Figure 8 shows the STEM images of $\left(\mathrm{A}^{\prime} \mathrm{A}^{\prime \prime}\right)_{2} \mathrm{BO}_{4}$-type $(\mathrm{La}, \mathrm{Sr})_{2} \mathrm{AlO}_{4},(\mathrm{La}, \mathrm{Sr})_{2} \mathrm{GaO}_{4}$, and $(\mathrm{Nd}, \mathrm{Ca})_{2} \mathrm{AlO}_{4}$ (see Supplementary Fig. 65 for composition verification of A-site solid solutions). As schematically depicted in the illustrations in the left-hand column, the A-site sublayers of these RP oxides encompass $[\mathrm{SrO}]^{0}\left(\right.$ or $\left.[\mathrm{CaO}]^{0}\right)$ with no local charge in addition to charged $[\mathrm{LaO}]^{+}\left(\right.$or $\left[\mathrm{NdO}^{+}\right)$as a solid solution. Consequently, the positive-charge electrostatics between the consecutive shear layers should diminish, resulting in comparatively weak interlayer repulsion. Considerably strong elongation of oxygen octahedra for screening the effective positive charge at the fault is thus no longer necessary. Indeed, as denoted in each of the magnified ABF images in the right-hand column, the length of $z$ axis Al-O and Ga-O bonds does not exceed $2.2 \AA$, in contrast to a value of $2.3 \AA$ in some nickelates (Fig. 6) and cobaltates (Fig. 7a) and even $2.5 \AA$ in cuprates (Fig. 7b). Therefore, this series of direct observations in Fig. 8 provides consistent evidence for our findings on the electrostatics-induced distortion of oxygen octahedral in RP oxides.

In conclusion, we have observed the formation of $[\mathrm{AX}]-[\mathrm{AX}]$ interleaved shear faults as common planar defects in five $\mathrm{ABX}_{3}$ perovskite-type phases, $\mathrm{SrTiO}_{3}, \mathrm{BaCeO}_{3}, \mathrm{LaCoO}_{3}, \mathrm{LaNiO}_{3}$, and $\mathrm{CsPbBr}$. As each fault generated in $\mathrm{LaCoO}_{3}$ and $\mathrm{LaNiO}_{3}$ consists of two positively charged $[\mathrm{LaO}]^{+}$sublayers in contrast to $[\mathrm{AX}]$ with no local charge in $\mathrm{SrTiO}_{3}, \mathrm{BaCeO}_{3}$, and $\mathrm{CsPbBr}$, strong electrostatic repulsion can be established at the fault plane. We identified that this charge-induced instability could remarkably diminish via the asymmetric tetragonal distortion of oxygen octahedra by the protrusion of oxygen anions toward the fault plane and thereby effectively screen the repulsion. Furthermore, via direct atomic-column-resolved visualization by $\mathrm{ABF}$ imaging in 14 representative RP oxides, our atomiclevel findings regarding the tetragonal distortion of oxygen octahedra at the shear faults provide crucial insight to consistently explain the octahedron elongation commonly observed in lanthanide-based RP oxides among more than 80 $\mathrm{RP}$-type oxides and halides. In addition to the electronic structure of metal cations on the basis of the Jahn-Teller effect, the present study suggests that the local electrostatic instability is a significant cause of the tetragonal distortion of oxygen octahedra in complex oxides.

\footnotetext{
Methods

Preparation of polycrystalline samples. To prepare polycrystalline perovskite and RP phase samples, powders were first synthesized via a conventional solid-state reaction method using $\mathrm{SrCO}_{3}$ (99.9\%, Sigma Aldrich) and $\mathrm{TiO}_{2}$ (99.9\%, Sigma Aldrich) for $\mathrm{SrTiO}_{3}, \mathrm{Sr}_{2} \mathrm{TiO}_{4}$, and $\mathrm{Sr}_{3} \mathrm{Ti}_{2} \mathrm{O}_{7} ; \mathrm{SrCO}_{3}$ and $\mathrm{SnO}_{2}$ (99.9\%, Sigma Aldrich) for $\mathrm{Sr}_{2} \mathrm{SnO}_{4}$; $\mathrm{BaCO}_{3}$ (99.999\%, Sigma Aldrich) and $\mathrm{CeO}_{2}$ (99.95\%, Sigma Aldrich) for $\mathrm{BaCeO}_{3}$; $\mathrm{La}(\mathrm{OH})_{3}\left(99.9 \%\right.$, Sigma Aldrich) and $\mathrm{NiO}\left(99.8 \%\right.$, Sigma Aldrich) for $\mathrm{La}_{3} \mathrm{Ni}_{2} \mathrm{O}_{7}$ and $\mathrm{La}_{4} \mathrm{Ni}_{3} \mathrm{O}_{10} ; \mathrm{La}(\mathrm{OH})_{3}$ and $\mathrm{Co}_{3} \mathrm{O}_{4}\left(99.5 \%\right.$, Sigma Aldrich) for $\mathrm{LaCoO}_{3} ; \mathrm{La}(\mathrm{OH})_{3}$ and $\mathrm{CuO}$ (99.99\%, Sigma Aldrich) for $\mathrm{La}_{2} \mathrm{CuO}_{4} ; \mathrm{Nd}_{2} \mathrm{O}_{3}$ (99.9\%, Sigma Aldrich) and $\mathrm{NiO}$ for $\mathrm{Nd}_{2} \mathrm{NiO}_{4}$; and $\mathrm{Pr}\left(\mathrm{NO}_{3}\right)_{3} \cdot 6 \mathrm{H}_{2} \mathrm{O}\left(99.9 \%\right.$, Sigma Aldrich) and $\mathrm{Ni}\left(\mathrm{CH}_{3} \mathrm{COO}\right)_{2} \cdot 4 \mathrm{H}_{2} \mathrm{O}(99 \%$, Sigma Aldrich) for $\mathrm{Pr}_{2} \mathrm{NiO}_{4}$. Each mixture of the starting materials with 5\%-excess A-site cations for perovskites and stoichiometric composition for RP phases was ballmilled in high-purity ethyl alcohol for $24 \mathrm{~h}$ with a zirconia jar and balls. After being dried, the slurries were calcined in air at $1100{ }^{\circ} \mathrm{C}$ for $10 \mathrm{~h}$ for $\mathrm{SrTiO}_{3}$; at $1200{ }^{\circ} \mathrm{C}$ for $10 \mathrm{~h}$ for $\mathrm{Sr}_{2} \mathrm{TiO}_{4}$ and $\mathrm{Sr}_{3} \mathrm{Ti}_{2} \mathrm{O}_{7}$; at $1000^{\circ} \mathrm{C}$ for $8 \mathrm{~h}$ for $\mathrm{Sr}_{2} \mathrm{SnO}_{4}$; at $1050^{\circ} \mathrm{C}$ for $10 \mathrm{~h}$ for $\mathrm{BaCeO}_{3}$; at $1050^{\circ} \mathrm{C}$ for $12 \mathrm{~h}$ for $\mathrm{LaCoO}_{3}$ and $\mathrm{La}_{2} \mathrm{CuO}_{4}$; at $1100^{\circ} \mathrm{C}$ for $12 \mathrm{~h}$ for $\mathrm{Nd}_{2} \mathrm{NiO}_{4}$ and $\mathrm{Pr}_{2} \mathrm{NiO}_{4}$; and at $1200{ }^{\circ} \mathrm{C}$ in an $\mathrm{O}_{2}$-flow atmosphere for $24 \mathrm{~h}$ for $\mathrm{La}_{3} \mathrm{Ni}_{2} \mathrm{O}_{7}$ for solidstate synthesis. For better sinterability and a dense microstructure, $10 \% \mathrm{Gd}$ was doped at the $\mathrm{Ce}$ sites, when polycrystalline $\mathrm{BaCeO}_{3}$ samples were prepared. The calcined
} 

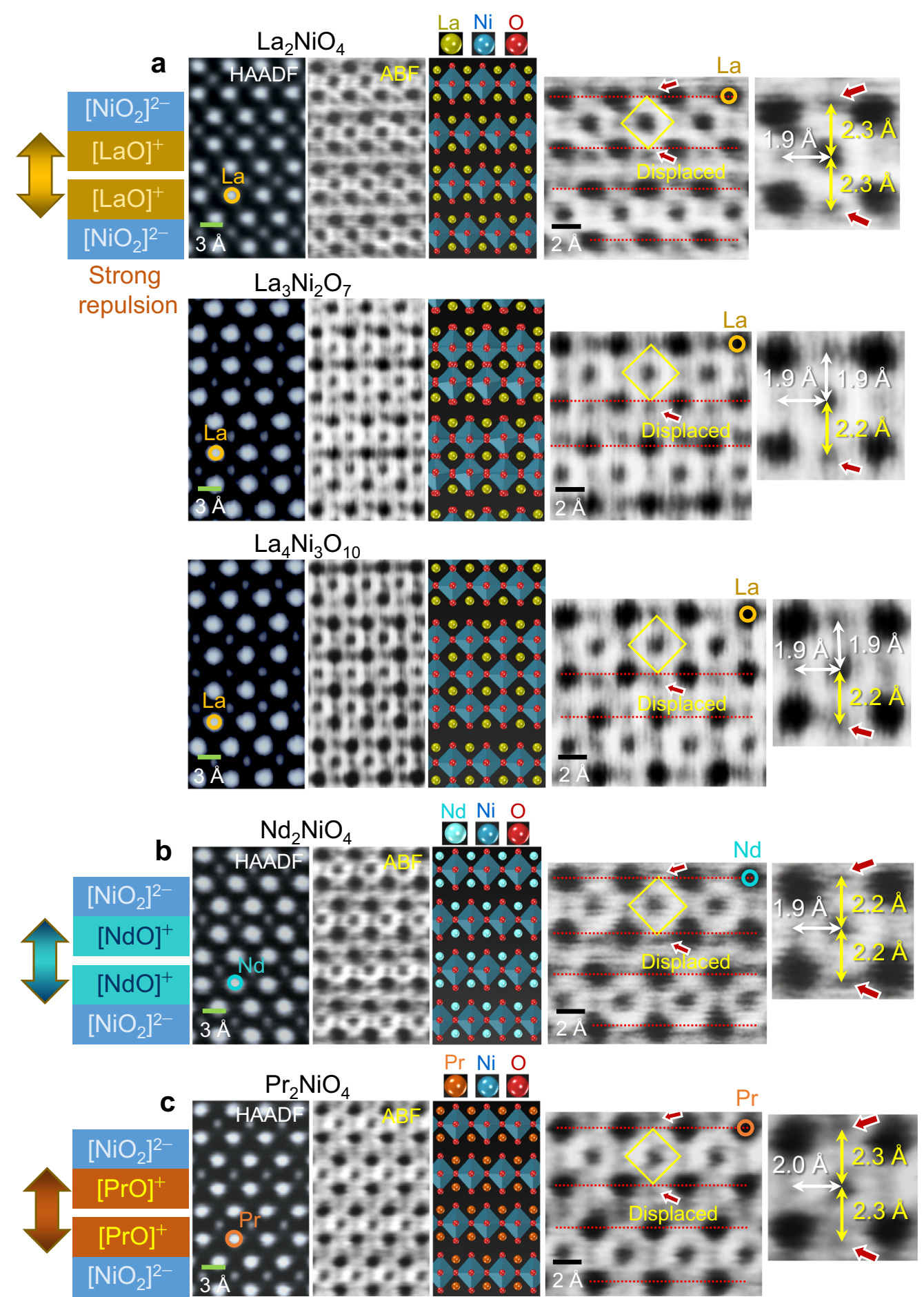

Fig. 6 STEM images showing the structure of lanthanide-based RP nickelates. Atomic-column-resolved images were acquired in a La $\mathrm{NiO}_{4}, \mathrm{La}_{3} \mathrm{Ni}_{2} \mathrm{O}_{7}$, and $\mathrm{La}_{4} \mathrm{Ni}_{3} \mathrm{O}_{10}, \mathbf{b ~} \mathrm{Nd}_{2} \mathrm{NiO}_{4}$, and $\mathbf{c} \mathrm{Pr}_{2} \mathrm{NiO}_{4}$, as listed in Supplementary Table 3. Strong local repulsion between [LnO] ${ }^{+}$sublayers is illustrated in the lefthand column. Significant elongation of the $\mathrm{Ni}-\mathrm{O}$ bonds at the shear planes is denoted by yellow arrows in the ABF images, providing quantitative information on the bond lengths. Red arrows in each image indicate substantial displacement of oxygen anions.

powders were ball-milled again to finally obtain fine particles. X-ray diffractometry (D/ Max-2500, Rigaku) with $\mathrm{Cu}-\mathrm{Ka}$ radiation confirmed that single-phase powders were synthesized. Each of the powders was slightly pressed into disks of $10 \mathrm{~mm}$ diameter and isostatically pressed under $200 \mathrm{MPa}$. The pellets were sintered in air at $1400{ }^{\circ} \mathrm{C}$ for $10 \mathrm{~h}$ for $\mathrm{SrTiO}_{3}, \mathrm{Sr}_{2} \mathrm{TiO}_{4}$, and $\mathrm{Sr}_{3} \mathrm{Ti}_{2} \mathrm{O}_{7}$; at $1550{ }^{\circ} \mathrm{C}$ for $12 \mathrm{~h}$ for $\mathrm{Sr}_{2} \mathrm{SnO}_{4}$; at $1400{ }^{\circ} \mathrm{C}$ for $5 \mathrm{~h}$ for $\mathrm{BaCeO}_{3}$ and $\mathrm{LaCoO}_{3}$; at $1300^{\circ} \mathrm{C}$ for $2 \mathrm{~h}$ for $\mathrm{La}_{2} \mathrm{CuO}_{4}$; at $1400^{\circ} \mathrm{C}$ for $2 \mathrm{~h}$ for $\mathrm{Nd}_{2} \mathrm{NiO}_{4}$ and $\mathrm{Pr}_{2} \mathrm{NiO}_{4}$; and at $1350^{\circ} \mathrm{C}$ for $2 \mathrm{~h}$ for $\mathrm{La}_{3} \mathrm{Ni}_{2} \mathrm{O}_{7}$ in an $\mathrm{O}_{2}$-flow atmosphere to obtain dense polycrystalline microstructure.
Thin-film fabrication. Epitaxial $\mathrm{LaNiO}_{3}, \mathrm{La}_{2} \mathrm{NiO}_{4}, \mathrm{La}_{2} \mathrm{CoO}_{4}$, and $\mathrm{La}_{4} \mathrm{Co}_{3} \mathrm{O}_{10}$ thin films were fabricated by using a sol-gel process. $\mathrm{La}\left(\mathrm{NO}_{3}\right)_{3} \cdot 6 \mathrm{H}_{2} \mathrm{O}(99.999 \%$, Sigma Aldrich) and $\mathrm{Ni}\left(\mathrm{CH}_{3} \mathrm{COO}\right)_{2} \cdot 4 \mathrm{H}_{2} \mathrm{O}\left(99.998 \%\right.$, Sigma Aldrich) for $\mathrm{LaNiO}_{3}$ and $\mathrm{La}_{2} \mathrm{NiO}_{4}, \mathrm{La}\left(\mathrm{NO}_{3}\right)_{3} \cdot 6 \mathrm{H}_{2} \mathrm{O}$ and $\mathrm{Co}\left(\mathrm{CH}_{3} \mathrm{COO}\right)_{2} \cdot 4 \mathrm{H}_{2} \mathrm{O}(99.999 \%$, Alfa Aesar) for $\mathrm{La}_{2} \mathrm{CoO}_{4}$ and $\mathrm{La}_{4} \mathrm{Co}_{3} \mathrm{O}_{10}$ were used as starting materials for the preparation of precursor solutions. They were dissolved in 2-methoxyethanol under a constant stirring condition to prepare precursor solutions with $0.2 \mathrm{M}$. To induce the favorable formation of shear faults, the La-excess nonstoichiometry was controlled 

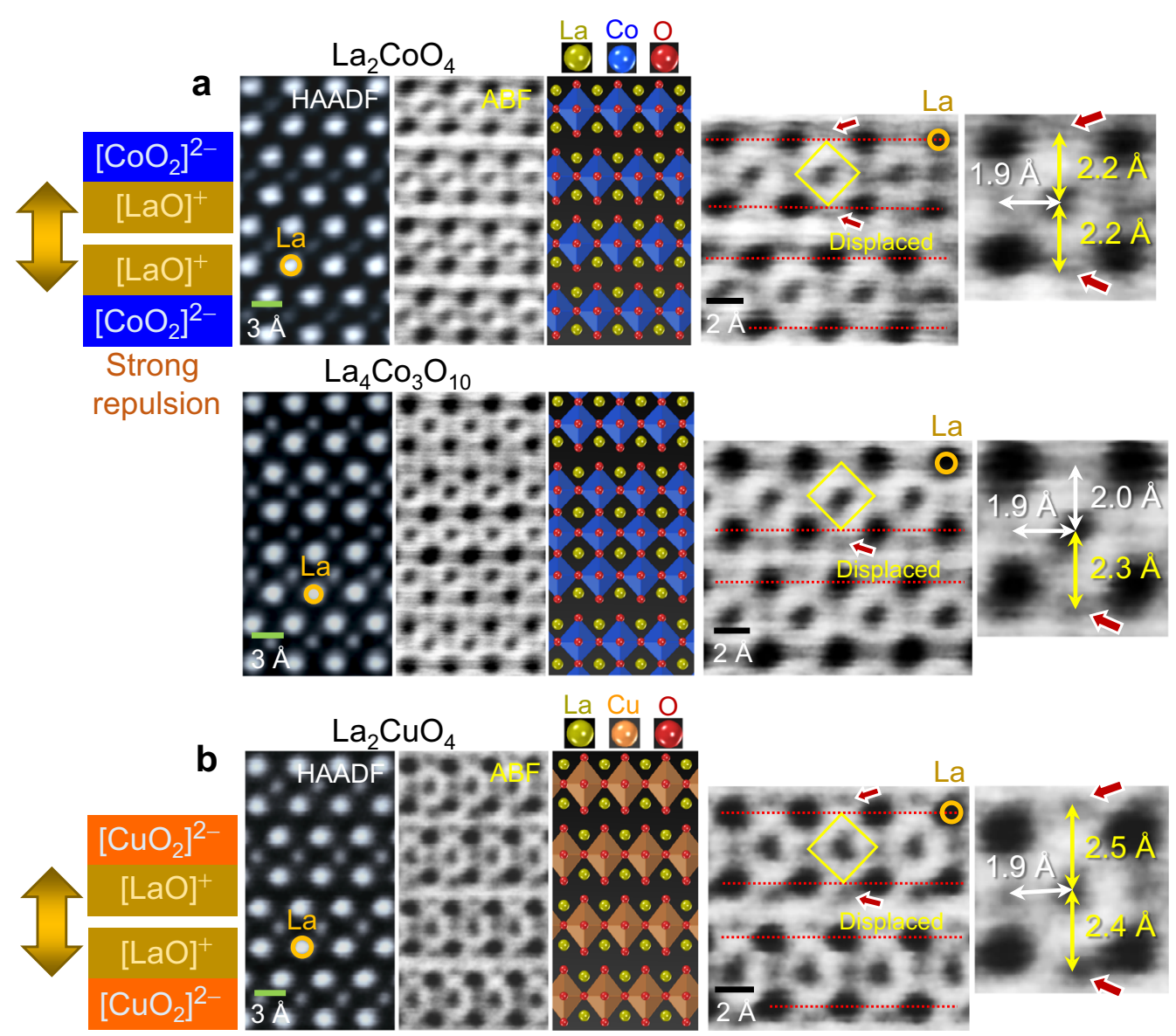

Fig. 7 STEM images showing the structure of La-based RP oxides. Atomic-column-resolved images were acquired in $\mathbf{a} \mathrm{La}_{2} \mathrm{CoO}_{4}$ and $\mathrm{La}_{4} \mathrm{CO}_{3} \mathrm{O}_{10}$ and $\mathbf{b}$ $\mathrm{La}_{2} \mathrm{CuO}_{4}$, as listed in Supplementary Table 3. Strong local repulsion between [LaO] ${ }^{+}$sublayers is illustrated in the left-hand column. Significant elongation of the $\mathrm{Co}-\mathrm{O}$ and $\mathrm{Cu}-\mathrm{O}$ bonds at the shear planes is denoted by yellow arrows in the ABF images, providing quantitative information on the bond lengths. As $\mathrm{Cu}^{2+}$ is known as a strong Jahn-Teller cation, $\left\{\mathrm{CuO}_{6}\right\}$ octahedra in $\mathrm{La}_{2} \mathrm{CuO}_{4}$ show more prominent $z$ axis elongation. Red arrows in each image indicate substantial displacement of oxygen anions.

by adding $3 \mathrm{~mol} \%$ more of the La nitrate for $\mathrm{LaNiO}_{3}$ films. For complete dissolution of the source materials, refluxing was carried out at $80^{\circ} \mathrm{C}$ for $1 \mathrm{~h}$. Each of the precursor solutions was deposited on (001) $\mathrm{LaAlO}_{3}$ and $(001) \mathrm{SrTiO}_{3}$ (Shinkosha, Japan) single-crystal substrates by a spin-coating method at $5000 \mathrm{rpm}$ for $10 \mathrm{~s}$. The wet films were dried at $150^{\circ} \mathrm{C}$ for $10 \mathrm{~min}$ on a hot plate, and then annealed at $800{ }^{\circ} \mathrm{C}$ for $1 \mathrm{~h}$ in the air for $\mathrm{LaNiO}_{3}$ films, at $900^{\circ} \mathrm{C}$ for $1 \mathrm{~h}$ in the air for $\mathrm{La}_{2} \mathrm{NiO}_{4}$ films, and at $1000^{\circ} \mathrm{C}$ for $1 \mathrm{~h}$ in $\mathrm{N}_{2}$-flow atmosphere for $\mathrm{La}_{2} \mathrm{CoO}_{4}$ and $\mathrm{La}_{4} \mathrm{Co}_{3} \mathrm{O}_{10}$ films for crystallization. X-ray diffractometry (X'Pert-PRO MRD, PANalytical) and STEM observation verified the epitaxy of grown films. Commercially available (001) single-crystal substrates (MTI corporation) were purchased for structure observation in $\mathrm{LaSrAlO}_{4}, \mathrm{LaSrGaO}_{4}$, and $\mathrm{NdCaAlO}_{4}$.

Nanocrystal synthesis. $\mathrm{Cs} \mathrm{PbBr}_{3}$ nanocrystals were synthesized by a microwaveassisted method ${ }^{50}$. A solvent mixture was prepared by using 1-octadecene $(5 \mathrm{~mL})$, oleylamine $(0.5 \mathrm{~mL})$, and oleic acid $(0.5 \mathrm{~mL})$. This mixture was degassed by evacuation and stirred in a round bottom flask at $170^{\circ} \mathrm{C}$ on a hot plate for $30 \mathrm{~min}$. After the degassing process, the mixture was transferred into a $10-\mathrm{mL}$ vial and followed by adding and dispersing $\mathrm{Cs}_{2} \mathrm{CO}_{3}(0.0217 \mathrm{~g}, 99.995 \%$, Sigma Aldrich) and $\mathrm{PbBr}_{2}(0.0738 \mathrm{~g}, 99.999 \%$, Sigma Aldrich) with a vortex mixer. This vial was placed into a microwave oven and subsequent microwave irradiation was carried out at $700 \mathrm{~W}$ for $5 \mathrm{~min}$. The 5 -min irradiation together with 1 -min intermission was further repeated eight more times, resulting in irradiation for $45 \mathrm{~min}$ in total. To separate residual solvents and a solid product, the mixture after the microwave irradiation was centrifuged at $1398 \times g$ for $5 \mathrm{~min}$. The solid product was dispersed in toluene and further purified by centrifugation at $5590 \times \mathrm{g}$ for $5 \mathrm{~min}$ so as to finally acquire a toluene solution containing homogeneously dispersed $\mathrm{CsPbBr}_{3}$ nanocrystals.

STEM, EELS, and specimen preparation. Specimens for STEM observation of sintered polycrystalline samples were prepared by mechanical grinding to a thickness of $80 \mu \mathrm{m}$, dimpling to a thickness of $<10 \mu \mathrm{m}$, and finally ion-beam thinning by using a precision ion polishing system (PIPS, Gatan Inc.) for electron transparency. STEM specimens for $\mathrm{LaNiO}_{3}$ and $\mathrm{NdNiO}_{3}$ thin films were prepared by lift out via ion-beam milling in a focused ion-beam system (Quanta 3D FEG and Helios G4 UX, ThermoFisher Scientific). Protective amorphous carbon and thin Pt layers were applied over the region of interest before milling. To minimize the sidewall damage and sufficiently thin the specimen for electron transparency, final milling was carried out at a voltage of $\sim 2 \mathrm{kV}$. HAADF- and ABF-STEM images were acquired with a transmission electron microscope (Titan cubed G2 60-300, ThermoFisher Scientific) at $200 \mathrm{kV}$ or $300 \mathrm{kV}$ with a spherical aberration (Cs) corrector (CEOS GmbH). The optimum size of the electron probe was $\sim 1 \AA$ with a convergence semiangle of $19 \mathrm{mrad}$. The collection semiangles of the STEM detectors were set to 40.0-200 mrad for HAADF imaging and 9.61-53.6 (or 10.1-19.1) mrad for ABF imaging. EELS analysis was performed with a Gatan Image Filter (GIF Quantum 965, Gatan Inc.). Electron energy-loss spectra for the Ba- $\mathrm{M}_{5}(781 \mathrm{eV})$ and $\mathrm{Ce}-\mathrm{M}_{5}(883 \mathrm{eV})$ edges were acquired for atomic-column spectrum imaging in $\mathrm{BaCeO}_{3}$ and with a dispersion of $0.25 \mathrm{eV}$ per channel and a collection aperture of $5 \mathrm{~mm}$ in diameter. The $\mathrm{O}-\mathrm{K}, \mathrm{Ti}-\mathrm{L}_{2,3}$, and $\mathrm{Co}-\mathrm{L}_{2,3}$ edges were also collected in $\mathrm{SrTiO}_{3}$ and $\mathrm{LaCoO}_{3}$ samples containing shear faults to examine whether the oxidation states of $\mathrm{Ti}$ and $\mathrm{Co}$ and the orbital hybridization with oxygen vary at the fault plane.

DFT calculations. Ab initio DFT calculations for DOS variation in $\mathrm{LaNiO}_{3}$ were carried out using the spin-polarized local density approximation (LDA) functional for exchange-correlation, along with the ultrasoft pseudopotentials for ionic cores, as implemented in the CASTEP code (Biovia Inc.). To consider the electron localization around $\mathrm{Ni}$ ions, the LDA $+\mathrm{U}$ method with the Hubbard $U$ parameter $(U=4.0 \mathrm{eV}$ for $\mathrm{Ni} 3 d$ states) was employed. A low-spin $\left(t_{2 g}{ }^{6}\right)\left(e_{g}\right)$ for $d^{7} \mathrm{Ni}^{3+}$ configuration was assumed for the spin-polarized calculations. The plane-wave basis set for the kinetic energy cutoff was $500 \mathrm{eV}$. Relaxation of the internal coordinates for each atom was performed using the Broyden-Fletcher-Goldfarb-Shanno (BFGS) algorithm with convergence tolerances of $0.1 \mathrm{eV} / \AA$ for the maximum ionic force, $5 \times 10^{-5} \mathrm{eV} /$ atom for the total energy, and $0.005 \AA$ for the maximum ionic displacement. 

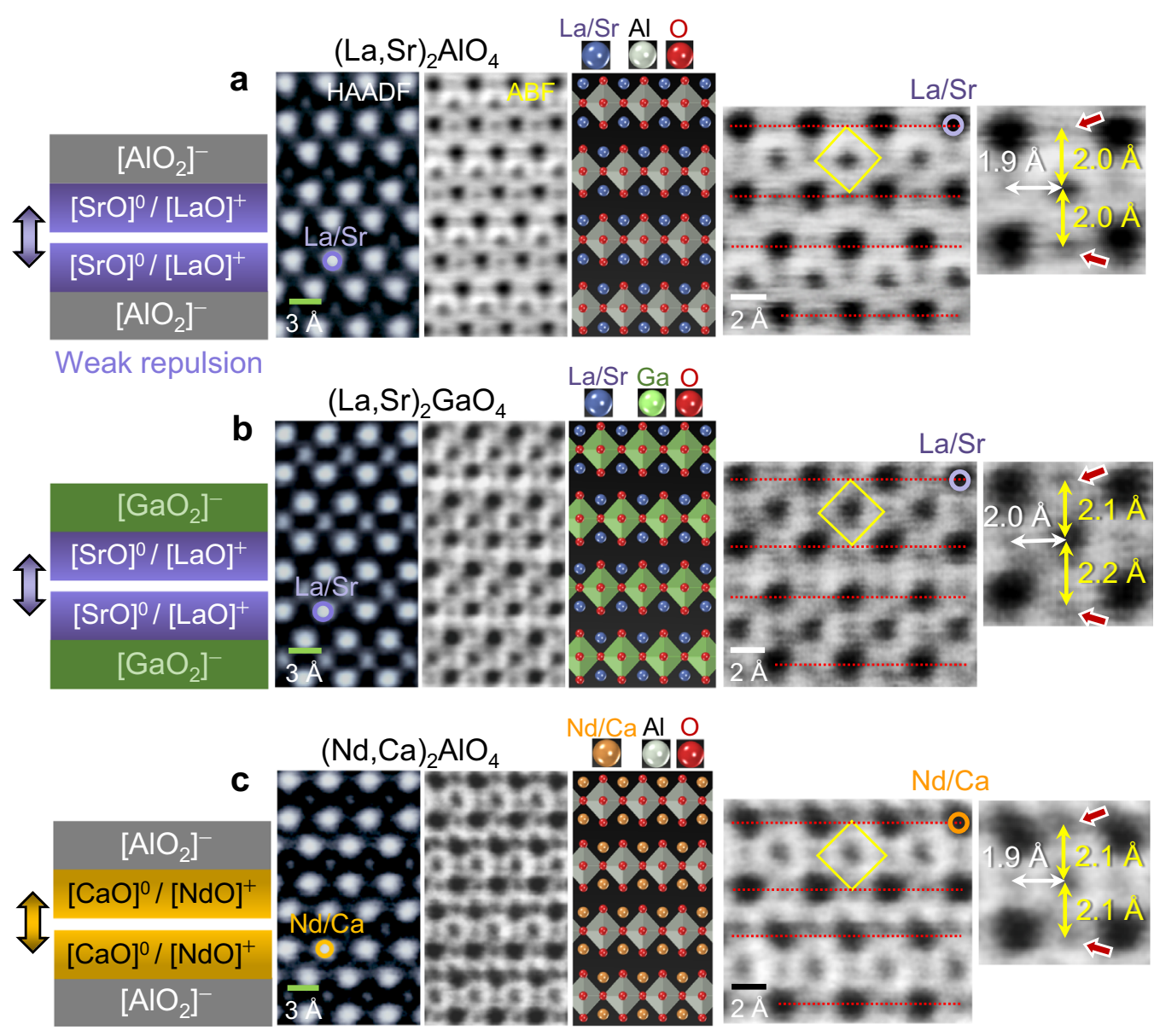

Fig. 8 STEM images showing the structure of $\left(\mathbf{A}^{\prime} \mathbf{A}^{\prime \prime}\right)_{2} \mathbf{B O}_{\mathbf{4}}$-type $\mathbf{R P}$ oxides. Atomic-column-resolved images were acquired in a $(\mathrm{La}, \mathrm{Sr})_{2} \mathrm{AlO}{ }_{4}, \mathbf{b}$ $(\mathrm{La}, \mathrm{Sr})_{2} \mathrm{GaO}_{4}$, and $\mathbf{c}(\mathrm{Nd}, \mathrm{Ca})_{2} \mathrm{AlO}_{4}$. Note that the $\mathrm{A}$-site sublayers include $[\mathrm{SrO}]^{0}$ (or $[\mathrm{CaO}]^{0}$ ) with no effective charge and subsequently the electrostatic repulsion between the layers diminishes. Therefore, the elongation of the $\mathrm{Al}-\mathrm{O}$ and $\mathrm{Ga}-\mathrm{O}$ bonds toward the shear planes is not remarkable, showing a bond length of $\leq 2.2 \AA$. Magnified ABF images directly provide quantitative information on the bond lengths. Red arrows in each image indicate a slight displacement of oxygen anions.

\section{Data availability}

The data sets generated during and/or analyzed during the current study are available from the corresponding author on reasonable request.

Received: 7 December 2020; Accepted: 3 September 2021; Published online: 20 September 2021

\section{References}

1. Pauling, L. The principles determining the structure of complex ionic crystals. J. Am. Chem. Soc. 51, 1010-1026 (1929).

2. Glazer, A. M. The classification of tilted octahedra in perovskites. Acta Crystallogr. B 28, 3384-3392 (1972).

3. Woodward, P. M. Octahedral tilting in perovskites. I. Geometrical considerations. Acta Crystallogr. B 53, 32-43 (1997).

4. Akamatsu, $\mathrm{H}$. et al. Inversion symmetry breaking by oxygen octahedral rotations in the Ruddlesden-Popper $\mathrm{NaRTiO}_{4}$ family. Phys. Rev. Lett. 112, 187602 (2014)

5. Pitcher, M. J. et al. Tilt engineering of spontaneous polarization and magnetization above $300 \mathrm{~K}$ in a bulk layered perovskite. Science $347,420-424$ (2015).

6. Kim, T. H. et al. Polar metals by geometric design. Nature 533, 68-72 (2016).

7. Lu, X.-Z. \& Rondinelli, J. M. Epitaxial-strain-induced polar-to-nonpolar transitions in layered oxides. Nat. Mater. 15, 951-955 (2016).

8. Zhu, T. et al. Cation exchange as a mechanism to engineer polarity in layered perovskites. Chem. Mater. 30, 8915-8924 (2018).
9. Yoshida, S. et al. Hybrid improper ferroelectricity in $(\mathrm{Sr}, \mathrm{Ca})_{3} \mathrm{Sn}_{2} \mathrm{O}_{7}$ and beyond: universal relationship between ferroelectric transition temperature and tolerance factor in $n=2$ Ruddlesden-Popper phases. J. Am. Chem. Soc. 140, 15690-15700 (2018).

10. Zhang, Y., Wang, J. \& Ghosez, P. Unraveling the suppression of oxygen octahedra rotations in $\mathrm{A}_{3} \mathrm{~B}_{2} \mathrm{O}_{7}$ Ruddlesden-Popper compounds: engineering multiferroicity and beyond. Phys. Rev. Lett. 125, 157601 (2020).

11. Bersuker, I. B. The Jahn-Teller Effect (Cambridge University Press, New York, 2006)

12. Halcrow, M. A. Jahn-Teller distortion in transition metal compounds, and their importance in functional molecular and inorganic materials. Chem. Soc. Rev. 42, 1784-1795 (2013).

13. Bak, J., Bae, H. B., Kim, J., Oh, J. \& Chung, S.-Y. Formation of twodimensional homologous faults and oxygen electrocatalytic activities in a perovskite nickelate. Nano Lett. 17, 3126-3132 (2017)

14. Wang, Z. et al. Dimensionality-driven insulator-metal transition in A-site excess non-stoichiometric perovskites. Nat. Commun. 1, 106 (2010).

15. Arredondo, M. et al. Chemistry of Ruddlesden-Popper planar faults at a ferroelectric-ferromagnet perovskite interface. J. Appl. Phys. 109, 084101 (2011).

16. Thind, A. S. et al. Atomic structure and electrical activity of grain boundaries and Ruddlesden-Popper faults in cesium lead bromide perovskite. Adv. Mater. 31, 1805047 (2019).

17. Guo, Q., Farokhipoor, S., Magén, C., Rivadulla, F. \& Noheda, B. Tunable resistivity exponents in the metallic phase of epitaxial nickelates. Nat. Commun. 11, 2949 (2020).

18. Bak, J., Bae, H. B., Oh, C., Son, J. \& Chung, S.-Y. Effect of lattice strain on the formation of Ruddlesden-Popper faults in heteroepitaxial $\mathrm{LaNiO}_{3}$ for oxygen evolution electrocatalysis. J. Phys. Chem. Lett. 11, 7253-7260 (2020). 
19. Choi, S.-Y., Chung, S.-Y., Yamamoto, T. \& Ikuhara, Y. Direct determination of dopant site selectivity in ordered perovskite $\mathrm{CaCu}_{3} \mathrm{Ti}_{4} \mathrm{O}_{12}$ polycrystals by aberration-corrected STEM. Adv. Mater. 21, 885-889 (2009).

20. Chung, S.-Y., Choi, S.-Y., Kim, T. H. \& Lee, S. Surface-orientation-dependent distribution of subsurface cation-exchange defects in olivine-phosphate nanocrystals. ACS Nano 9, 850-859 (2015).

21. Yoon, H.-I. et al. Probing dopant segregation in distinct cation sites at perovskite oxide polycrystal interfaces. Nat. Commun. 8, 1417 (2017).

22. Ishikawa, R. et al. Direct imaging of hydrogen-atom columns in a crystal by annular bright-field electron microscopy. Nat. Mater. 10, 278-281 (2011).

23. Findlay, S. D., Azuma, S., Shibata, N., Okunishi, E. \& Ikuhara, Y. Direct oxygen imaging within a ceramic interface with some observations upon the dark contrast at the grain boundary. Ultramicroscopy 111, 285-289 (2011).

24. Kim, H.-S., Jang, A., Choi, S.-Y., Jung, W. \& Chung, S.-Y. Vacancy-induced electronic structure variation of acceptors and correlation with proton conduction in perovskite oxides. Angew. Chem. Int. Ed. 55, 13499-13503 (2016).

25. Kim, J. H. et al. Atomic-scale observation of oxygen substitution and its correlation with hole-transport barriers in $\mathrm{Cu}_{2} \mathrm{ZnSnSe}_{4}$ thin-film solar cells. Adv. Energy Mater. 6, 1501902 (2016).

26. Byeon, P. et al. Atomic-scale observation of $\mathrm{LiFePO}_{4}$ and $\mathrm{LiCoO}_{2}$ dissolution behavior in aqueous solutions. Adv. Funct. Mater. 28, 1804564 (2018).

27. Heo, Y. et al. Symmetry-broken atomic configurations at grain boundaries and oxygen evolution electrocatalysis in perovskite oxides. Adv. Energy Mater. 8, 1802481 (2018).

28. Bak, J., Bae, H. B. \& Chung, S.-Y. Atomic-scale perturbation of oxygen octahedra via surface ion exchange in perovskite nickelates boosts water oxidation. Nat. Commun. 10, 2713 (2019).

29. Byeon, P. et al. Atomic-scale unveiling multiphase evolution during hydrates Zn-ion insertion in vanadium oxide. Nat. Commun. 12, 4599 (2021).

30. Ohtomo, A. \& Hwang, H. Y. A high-mobility electron gas at the $\mathrm{LaAlO}_{3} /$ $\mathrm{SrTiO}_{3}$ heterointerface. Nature 427, 423-426 (2004).

31. Nakagawa, N., Hwang, H. Y. \& Muller, D. A. Why some interfaces cannot be sharp. Nat. Mater. 5, 204-209 (2006).

32. Wang, L. et al. Strain Effect on oxygen evolution reaction activity of epitaxial $\mathrm{NdNiO}_{3}$ thin films. ACS Appl. Mater. Interfaces 11, 12941-12947 (2019).

33. Bak, J., Heo, Y., Yun, T. G. \& Chung, S.-Y. Atomic-level manipulations in oxide and alloys for electrocatalysis of oxygen evolution and reduction. ACS Nano 14, 14323-14354 (2020).

34. Shah, A. B. et al. Probing interfacial electronic structures in atomic layer $\mathrm{LaMnO}_{3}$ and $\mathrm{SrTiO}_{3}$ superlattices. Adv. Mater. 22, 1156-1160 (2010).

35. Gazquez, J. et al. Atomic-resolution imaging of spin-state superlattices in nanopockets within cobaltite thin films. Nano Lett. 11, 973-976 (2011).

36. Zhu, G.-Z., Radtke, G. \& Botton, G. A. Bonding and structure of a reconstructed (001) surface of $\mathrm{SrTiO}_{3}$ from TEM. Nature 490, 384-387 (2012).

37. Song, C. W., Suh, H., Bak, J., Bae, H. B. \& Chung, S.-Y. Dissolution-induced surface roughening and oxygen evolution electrocatalysis of alkaline-earth iridates in acid. Chem. 5, 3243-3259 (2019).

38. Shukla, A. K. et al. Unravelling structural ambiguities in lithium- and manganese-rich transition metal oxides. Nat. Commun. 6, 8711 (2015).

39. Li, L. et al. Hidden subsurface reconstruction and its atomic origins in layered oxide cathodes. Nano Lett. 20, 2756-2762 (2020).

40. Lee, C.-H. et al. Exploiting dimensionality and defect mitigation to create tunable microwave dielectrics. Nature 502, 532-536 (2013).

41. Lee, Y.-L. et al. Kinetics of oxygen surface exchange on epitaxial Ruddlesden-Popper phases and correlations to first-principles descriptors. J. Phys. Chem. Lett. 7, 244-249 (2016).

42. Gu, X.-K. \& Nikolla, E. Design of Ruddlesden-Popper oxides with optimal surface oxygen exchange properties for oxygen reduction and evolution. ACS Catal. 7, 5912-5920 (2017).

43. Meyer, T. L. et al. Strain control of oxygen kinetics in the Ruddlesden-Popper oxide $\mathrm{La}_{1.85} \mathrm{Sr}_{0.15} \mathrm{CuO}_{4}$. Nat. Commun. 9, 92 (2018).

44. Zhu, Y. et al. Unusual synergistic effect in layered Ruddlesden-Popper oxide enables ultrafast hydrogen evolution. Nat. Commun. 10, 149 (2019).

45. Liu, S., Sun, C., Chen, J., Xiao, J. \& Luo, J.-L. A high-performance Ruddlesden-Popper perovskite for bifunctional oxygen electrocatalysis. ACS Catal. 10, 13437-13444 (2020).
46. Cohen, R. E. Origin of ferroelectricity in perovskite oxides. Nature 358, 136-138 (1992).

47. Bersuker, I. B. Pseudo-Jahn-Teller effect-a two-state paradigm in formation, deformation, and transformation of molecular systems and solids. Chem. Rev. 113, 1351-1390 (2013).

48. Akamatsu, H. et al. A-site cation size effect on oxygen octahedral rotations in acentric Ruddlesden-Popper alkali rare-earth titanates. Phys. Rev. Mater. 3, 065001 (2019).

49. Balachandran, P. V., Puggioni, D. \& Rondinelli, J. M. Crystal-chemistry guidelines for noncentrosymmetric $\mathrm{A}_{2} \mathrm{BO}_{4}$ Ruddlesden-Popper oxides. Inorg. Chem. 53, 336-348 (2014).

50. Long, Z., Ren, H., Sun, J., Ouyang, J. \& Na, N. High-throughput and tunable synthesis of colloidal $\mathrm{CsPbX}_{3}$ perovskite nanocrystals in a heterogeneous system by microwave irradiation. Chem. Commun. 53, 9914-9917 (2017).

\section{Acknowledgements}

This work was supported by the National Research Foundation of Korea (NRF), grant numbers 2021M3H4A1A01079300 and 2021R1A2C2006072. S.-Y.C. was also financially supported by the Samsung Research Funding Centre of Samsung Electronics, grant no. SRFC-MA1602-52.

\section{Author contributions}

S.-Y.C. conceived and initiated the project, carried out the DFT calculations, assisted the STEM analyses, and wrote the paper. Y.Hong carried out all the syntheses and specimen preparation, performed the STEM image analyses, and collected the structure data of oxides. P.B. and H.B.B. performed the STEM, EELS and EDS analyses and contributed to the acquisition of image data. J.B., Y.Heo and H.-S.K. supported the experiments and specimen preparation. All the authors discussed the results and commented on the manuscript.

\section{Competing interests}

The authors declare no competing interests.

\section{Additional information}

Supplementary information The online version contains supplementary material available at https://doi.org/10.1038/s41467-021-25889-6.

Correspondence and requests for materials should be addressed to Sung-Yoon Chung

Peer review information Nature Communications thanks Rohan Mishra and the other, anonymous, reviewer(s) for their contribution to the peer review of this work.

Reprints and permission information is available at http://www.nature.com/reprints

Publisher's note Springer Nature remains neutral with regard to jurisdictional claims in published maps and institutional affiliations.

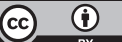

Open Access This article is licensed under a Creative Commons Attribution 4.0 International License, which permits use, sharing, adaptation, distribution and reproduction in any medium or format, as long as you give appropriate credit to the original author(s) and the source, provide a link to the Creative Commons license, and indicate if changes were made. The images or other third party material in this article are included in the article's Creative Commons license, unless indicated otherwise in a credit line to the material. If material is not included in the article's Creative Commons license and your intended use is not permitted by statutory regulation or exceeds the permitted use, you will need to obtain permission directly from the copyright holder. To view a copy of this license, visit http://creativecommons.org/ licenses/by/4.0/.

(C) The Author(s) 2021 